\title{
Global Network Structures and Problems of Aggregated Evaluation
}

\author{
Dmytro Polishchuk ${ }^{1, a}$, Olexandr Polishchuk ${ }^{1, b,{ }^{*}}$
}

\author{
${ }^{1}$ Department of Nonlinear Mathematical Analysis, Pidstryhach Institute for Applied Problems of \\ Mechanics and Mathematics, National Academy of Sciences of Ukraine, Lviv, Ukraine
}

ad_pole@mail.ru, bod_polishchuk@ukr.net

\begin{abstract}
Keywords: Complex System, Network, Multiplex, Association, Conglomerate, Continuous Monitoring, Big Data, Aggregation, Evaluation
\end{abstract}

\begin{abstract}
This article covers the problem of processing of Big Data that describe process of complex networks and network systems operation. It also introduces the notion of hierarchical network systems combination into associations and conglomerates alongside with complex networks combination into multiplexes. The analysis is provided for methods of global network structures study depending on the purpose of the research. Also the main types of information flows in complex hierarchical network systems being the basic components of associations and conglomerates are covered. The main approaches for the formation of generalized conclusions about operation quality of complex hierarchical network systems are analyzed. Advantages and drawbacks of the "weakest" element method and a weighted linear aggregation method are determined. Nonlinear aggregation method is proposed for evaluating the quality of the system, which consists of elements of the same priority. Hybrid approaches to form generalized conclusions are developed based on the main aggregation methods. It is shown that they allow to obtain more reliable aggregation results.
\end{abstract}

\section{Introduction}

Complex large scale technological systems (CLSTS) are used almost in all areas of human activity, e.g. in transportation (railway, road and aviation systems, transportation networks of large cities and regions of countries) [1], supply and logistics (systems for power, gas, petrol, heat and water supply, trade networks) [2], information and communication (Internet, TV, radio, post service, press, fixed and mobile telephony) [3], in economics (networks of state-owned and (or) private companies, their suppliers and final products distributors) [4], finance (banking and insurance networks, money transfer systems) [5], education, healthcare etc. Their state and operation quality impose large impact on citizens' quality of life, efficiency of economy and possibilities for its development, as well as government structures readiness to mitigate impacts of technological and natural disasters. Finally, they may be treated as the evidences of country development level in general $[6,7]$. Failure of one of the elements of such system can often lead to operation breakdown or destabilization of the whole CLSTS. The example of this is cascading phenomenon [8]. Often the situations of the kind (e.g. accidents at nuclear or large chemical plants and other hazardous facilities, power lines, gas pipelines etc.) may lead to harsh consequences, such as environmental disasters, property loss and numerous human victims [9]. These circumstances determine the importance of continuous monitoring of technological systems operation, careful control of their behavior and timely response to emerging threats [10,11]. These circumstances determine the importance of continuous monitoring of technological systems operation, careful control of their behavior and timely response to emerging threats. Solution for this problem belongs to the fields of systems theory, system analysis, complex networks theory, mathematical modelling etc [12-14]. Every CLSTS is a complex dynamical system (CDS). It is usually difficult to implement classical mathematical modelling methods on practice for studying most existing CDS due to the problems of dimension and adequacy. Therefore, decomposition and investigation of the properties of the separate components of the system is the usual method for modeling. However, the important features of the interaction between components of CDS may be lost during decomposition. A lot of problems arise when trying to optimize the complex large scale systems [15]. 
Optimizing separate components does not always guarantee the quality improvement of the whole system, and synchronous optimization of all components of CDS is usually unrealistic goal (hard to imagine the simultaneous repair of all roads of megapolis). Network analysis methods [13, 16] are focused mostly on studying of system structure and interconnections between network elements without analysis of their state and functioning quality. At the same time, flow processing in the node may be quite a complicated process [17]. System analysis in general is aimed on selection of alternatives about further actions with respect to real CDS (development plans, ways to optimize, etc.) [12]. Taking into account the human factor is a separate problem in systems research. Often the influence of this factor can not account for any mathematical methods.

Complex systems appear, operate and develop within long periods of time and with natural processes of "aging", despite regular improvements, more strict and accurate control over their behaviour is required. This is why the development of methods for evaluation and forecasting the state, operation quality and interaction between structural elements of CLSTS is actual problem [1822]. Often the cause of accidents is the wrong evaluation of the current situation or an inadequate forecast of its future development. Sometimes the reason of accidents become "unfavorable coincidence of many random unlikely circumstances". Scientific discipline is not created yet, which could mathematically formalize such causes and forestall the disasters (accidents on the railway, nuclear power plants, hazardous industries, etc.). Catastrophe theory [23], which includes the bifurcation theory of dynamical systems (differential equations) and the theory of singularities of smooth mappings, is still far from solving the problems of real systems. Evaluation theory allows to determine the preconditions that can lead to catastrophic events (deterioration of the state or operation quality of system components, the weakening of the interaction between them, etc.). It also allows to determine the elements that threaten or require urgent optimization, and to analyze the influence of this optimization on the other system components and the whole CLSTS.

Multi-criteria and multi-parameter analysis of the state and operation quality of the system elements leads to a huge number of local evaluations. Manual analysis of such amounts of data to make correct and timely decisions is practically impossible. Therefore, a special place in evaluation theory take methods for formation of generalized (aggregated) conclusions.

Aggregation of evaluations of system components is known for a long time [22, 24, 25]. We used it to make generalized conclusions about the behavior of system elements according to the set of characteristics, parameters and evaluation criteria [26, 27] in a continuous, discrete, conceptual, and precise rating scale $[21,22]$. Aggregation is useful for determining the optimal operation modes of CDS [28] and selecting an optimal system from a given class of equivalent systems [29]. It can be used to analyze the history of CDS functioning and forecasting its behavior [21, 30].

As a rule, the weighted linear aggregation method (WLAM) is used to form generalized conclusions about the state and operation quality of the system [24-27]. In this paper we analyze three approaches to form aggregated evaluations: the "weakest" element method (WEM), WLAM and nonlinear aggregation method (NAM). We determine the relationship between the evaluations obtained by these methods and propose hybrid approaches for aggregated evaluation of systems that increase adequacy of generalized conclusions.

\section{Complex Networks, Network Systems, Multiplexes, Associations and Conglomerates}

During recent years, the theory of complex networks has been rapidly developing [13, 31]. We encounter network structures [32, 33] while studying micro- (e.g. quantum networks of fermions connected with bosons) and macroworld (gas networks in Universe, networks of black holes etc.). They occur in nature (e.g. protein and metabolism networks) and human society (e.g. Internet, language and citation networks). Complex technology systems (transportation, trade and power supply networks etc.) are not exceptions either. In general, an arbitrary network is defined as a statistical assembly, i.e. a set of networks with each network having certain probability of implementation, or as a set of all possible conditions of the given network. On the other hand, complex networks are graphs, i.e. the sets of nodes connected by some relations with nontrivial topological properties. When talking about real networks these properties determine network operation features [34]. 
Nodes of one network may be the nodes of many other networks at the same time. Thus, every town in the country can be a node for several transportation networks, as well as state and local administration networks, economy and financial network etc. Every person is also the node of many networks (family, professional, social etc.). Combination of several networks with non-empty intersection of nodes is called a multiplex [35]. Each network being the component of multiplex is called a layer. Examples above show that there are different types of interactions between the nodes existing on different layers of multiplex. These interactions may be of various nature or meaning and may have different material media.

Sometimes complex networks are called "the systems". In our opinion, network only reflects the structure of a system being its frame. There are flows that move along the network that make it a system. Real networks are created and exist with an aim to arrange the flows of certain type. These flows can be continuous (e.g. power resources), discrete (e.g. trains) or continuous-discrete (phone calls). The motion of flows in the network can be ordered (railway traffic), partially ordered (car traffic in large cities) or unordered (information flows in social networks). Networks with different types and levels of flow arrangement are generated different network systems [36]. Flow properties of certain network allow to divide multiplexes into network layers in a proper way.

CLSTS complies with any definition of a complex network and even network system only to some extent. The reason is that flows movement in majority of artificial technology networks need to be supported on organizational level. This function is performed by CLSTS control system which has hierarchical structure. Hierarchical network structures (HNS) are special because each subsystem of a certain hierarchy level consists of a set of subsystems which form subnetwork of lower hierarchy level network (see Fig. 1). Flow movement for which the network was created is performed at network of the lowest level. At the higher (control) levels, flows are represented by information, organizational and administrative decisions etc. HNS differs from common three dimensional tree structure by links between the nodes of each hierarchy level.

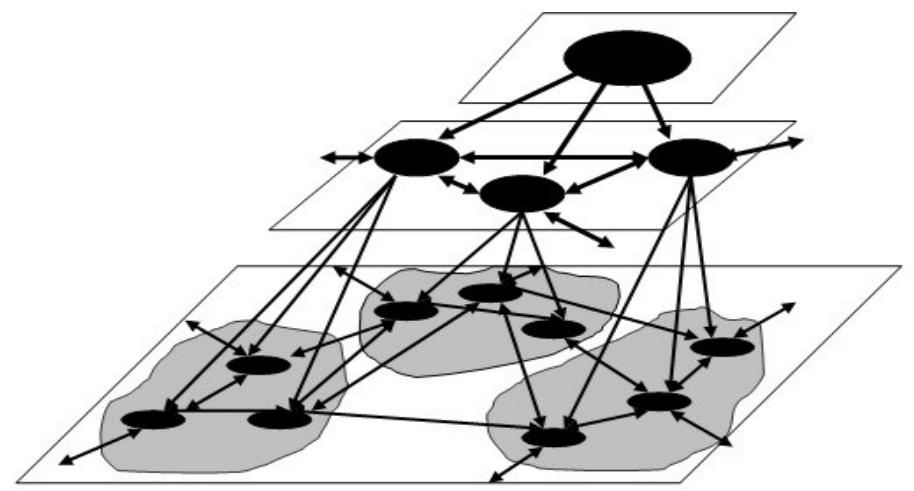

Fig. 1. Hierarchical network structure

There is an alternative to multiplex method of complex network systems combination. It consists in their joint engagement for solving important social or economical problems, e.g. industrial or natural disasters, pandemics, acts of terrorism etc. Solving these problems requires the interaction of many systems of different type and purpose: rescue and fire services, police, security agencies, military and medical units etc. The structures of the kind often arise in the industry, politics, social life etc. We call supersystems resulting from diverse complex hierarchical network systems (CHNS) interaction "conglomerates". Conglomerates often play more important role than multiplexes. Effective operation of conglomerates requires timely information exchange between their components. First of all, this means that interaction between CHNSs composing conglomerate has to be very tight. Relations between conglomerate components get less or more tighter depending on the purpose of their interaction and the extent of its implementation. Existence of purpose of creation and specific nature of relations are another aspects that make this structure different from multiplex. 
The components of the conglomerate can comprise combination of several interacting systems of the same type. We call the structures of the kind "associations". Signs of uniformity determine whether some formation is a conglomerate or an association. If we talk about economy of the country in general, the combination of all transportation networks can be considered an association, since in this case the sign of uniformity is represented by the purpose of such system operation which lies in passengers and cargo transportation. At the same time, transportation system of the country is conglomerate of transportation systems of different types. Associations among components of such conglomerates are presented by transport operators organizations, transportation companies etc.

Sometimes the dilemma arises of whether some formation shall be considered a multiplex or a conglomerate. In general, it depends on purpose and extent of the study. Network of towns within the country is the basis for organization of flows of various types. From this point of view, it can be considered multiplex each layer of which provides a different type of flow movement (rail, road, air, sea, river), and each node of multiplex can support movement of up to all possible types of flows. However, the cooperation of transport systems of various types with an aim to transport passengers and cargo inside and outside the country can be considered conglomerate. Thus, the transportation system of the country can be considered multiplex, conglomerate or association within the larger conglomerate (for example, industrial) depending on the purpose of the study. Note that during the study of complex networks and multiplexes, structure properties have higher priority, and during the study of network systems, associations and conglomerates, the most important is the function implementing the purpose of their creation.

In general, the modern world is a huge dynamic multiplex-conglomerate structure with thousands of systems and billions of elements (nodes). Complex study of real multiplexes and conglomerates that constitute components of this structure requires complete and comprehensive understanding of their state and operation even in the case of their decomposition into network layers, associations and separate network systems. Such understanding can be achieved with the use of information about the history, current condition and forecast of systems behavior. The volume of this information, its diversity and problem of timely information flows content analysis lead to emergence of the phenomenon called Big Data.

\section{Big Data and Information Overload}

Big Data is the series of approaches and tools for processing huge volumes of structured and unstructured data. Properties of Big Data is defined by "three V": volume (large volume of data), variety (diversity of data), and velocity (processing speed and efficiency of results obtaining) [37]. The main goal of Big Data processing is to achieve the results that can be perceived by human. Before making decision, people try to get information that provides comprehensive characteristics of the problem being solved. However, as well as the lack of information, the excess of it can lead to achieving wrong results. Such situation is called information overload or the problem of analysis paralysis. It applies to CLSTS as well. In 1995, the study was carried out in the USA [38] with an aim to calculate the number of documents being processed by the employees of three large corporations. The result achieved was between 35 and 64 thousands. 20 years passed since that time. However, it is unlikely that information load on people working in those corporations, as well as other ones, has decreased since the volume and the number of information flows rise every year. It is important that the person responsible for operation of critical and dangerous facilities does not get lost in the flow of data describing the operation process of those facilities. Their task is to timely localize and solve the problem. To overcome information overload it is necessary to extract only the information essential for making correct and timely decision. Indeed, only small amount of data available to the person making decision satisfies this requirement. For example, the cracks in the railway rail have often led to accidents with numerous victims. The railway detector carriage used for cracks detection extracts the data with $1 \mathrm{~mm}$ step (in many countries, railroads are tens and hundreds of thousands kilometers long). As the result, multibillion arrays of numerical data [39] are obtained. At the same time, the only thing end user (maintenance unit) must know is the exact 
location of the crack. Many examples alike may be provided from power industry, economy and finance, medicine etc. The data regarding system elements behavior may continuously come in huge amounts from many sources and require real time processing. It is often necessary to store these data for long and short term CLSTS state and behavior forecasting. Many systems are sensitive to small changes the accumulation of which can be a threat to their normal operation. Analysis of stored information allows to identify the negative phenomena trends in advance and to prevent them beforehand.

\section{Information Flows in Complex Hierarchical Network Systems}

We consider conglomerates as the combination of CLSTSs that interact to achieve common goals. Some CLSTSs can be grouped into associations according to uniformity. Every CLSTS is a complex hierarchical network system. At each level of the hierarchy, the edges ensure smooth motion of flows of certain type, whereas the nodes ensure their processing. Under the component of the system we understand its structural unit of any hierarchy level from element to subsystem of the highest decomposition level. Subsystems of the lowest decomposition level that consist of elements will be regarded to as the "basic subsystems" (BSS). The principles of the complex hierarchical network systems operation and methods of their behavior analysis are described in detail in [1].

In CHNS three main types of flows are distinguished (see Fig. 2):

1) ascending flows which come from controlled components to control components and may contain either processed primary data or aggregated data;

2) descending flows which come from control components to controlled components and contain information necessary for normal operation of controlled components, as well as decisions regarding their further actions;

3) network (horizontal) flows that come from some network components of certain hierarchy level to other components of the same hierarchy level.

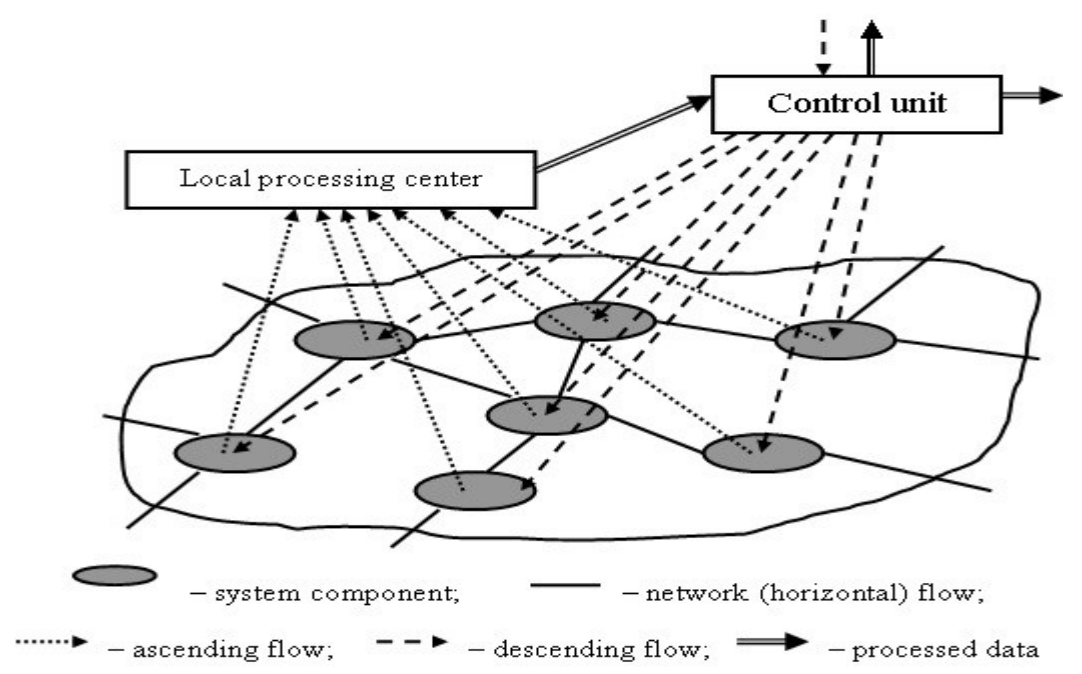

Fig. 2. Subsystem of CHNS

In general, ascending and descending flows implement cyclic (reverse) connection between control and controlled system components. These flows facilitate making correct and timely decisions regarding CLSTS operation process. Network flows provide self-organization of this process at every hierarchy level. These are the messages from one station regarding train delay to other stations located on the route of this train. Another example of such flows is professional information exchange in a team working on some project. In addition to the above mentioned, there are flows which run between the interacting systems of association or conglomerate. That is, we can add the intersystem flows to the listed intrasystem flows. One of the most important problems of CLSTS information support is synchronization of data flows between different CHNS hierarchy levels, within the networks of each hierarchy level and between the components of association or conglomerate. 
We can also distinguish different levels of information processing. The first (the lowest) level consists in continuous extracting and real time processing of large volumes of numerical data that simultaneously arrive from many network elements (nodes) to local data processing and control centers. At this hierarchy level (level of system BSS) data analysis and timely response to detected local problems in the network elements (nodes, edges and flows) is carried out. The main purpose of information analysis at this level is to discover potentially failure (or "the weakest") BSS elements. Failure of such elements often leads to the cascading phenomena (all catastrophes usually start from "minor failures" at system elements level). In such cases response time is major factor that allows to timely localize the problem and quickly overcome its consequences. Here it is expedient not only to determine current state but also to predict occurrence of potentially failure elements on the basis of previous evaluations history. In case such elements are missing, information processing center prepares the aggregated reports regarding condition of BSS elements and BSS in general for relevant control units. These reports are used as the basis for making objective and reasoned decision regarding further actions on this BSS. On this level, data on noncritical negative (which over time tends to become critical one) are accumulated for submission to upper control levels with an aim to solve existing problems and to prevent potential ones. In fact, at this level, objective information used to support decision-making by the control system units of the highest hierarchy levels shall be extracted and ordered.

Higher hierarchy levels receive the data aggregated in different ways [40]. Basic requirements for this data are as follows:

1) objectivity, i.e. they shall be based on reliable information only;

2) comprehensive description of the situation;

3) minimum sufficiency, i.e. the absence of duplicate and unimportant data;

4) simplicity and understandability, i.e. visualization methods shall be chosen that allow to quickly orient in the huge amount of conclusions obtained.

\section{Evaluation of Complex Hierarchical Network Systems}

The most of created and controlled by a man industrial, transportation, financial and other systems are the complex hierarchical network systems. Methods for complex evaluation of the state, operation quality and interaction between components of CHNS were described in [36]. These methods detemine the way for reflecting CHNS experimental studies data onto structured, according to hierarchy, sequence of local, forecasting, interactive and generalized conclusions about system behaviour. Taking into account the diversity of CHNS objects, these methods defines universal principles of such evaluations development, common for all objects of the same type and functional destination considering peculiarities of the former.

Evaluation of real systems is reasonable to start with objects of lowest structural level, i.e. with their elements. We define an element as an object of clearly defined location, functional destination and relevant set of characteristics describing its state and functioning process with corresponding ranges of permissible values for those characteristics. All characteristics are evaluated according to certain collection of criteria and parameters [22]. Of course, evaluation of every object presupposes evaluation of its state on the first place, and only after that the evaluation of quality of implementation of its functions that in any case depend on element's state - either directly or indirectly. The process of evaluation is started only after the stage of thorough extraction and processing of experimental data as to each of characteristic and their conversation into format, suitable for further analysis. Evaluations for elements' state and functions they implement on the basis of their characteristics behaviour analysis we call local [30].

Scheduled inspections of system's components are held at different time points, which means the results of last study may not stay on such stage till following inspection, and state of component and its operation quality may cross "safety threshold" [41]. It should be also taken into account that every real system evolves in time, i.e. with regard to current requirements, its evaluation may be insufficient. Therefore, evaluation process should contain means of analysis of CDS's meeting expected requirements for short- and long-term perspective. Thus, the evaluation process should not 
only determine conclusions and discover "faulty" elements for the time point moment when study is held, but also it should forecast further behaviour of system components. Forecasting analysis performed on the basis of local evaluations prehistory, allows us to determine the nature, direction and speed of system state change, follow up negative processes and forecast potential risks, as well as material and financial expenses required for their elimination or timely prevention [30].

Due to the number of reasons, scheduled inspections may often not discover drawbacks that arise "out of schedule". It should be also taken into account that even excellent state and functioning quality of separate components in the system do not ensure high performance of its subsystems or system in general. And vice versa, the most optimal work organization process will not ensure high efficiency of system functioning if CHNS's state or organization of components functioning is unsatisfactory. The more worn-out CHNS's objects are the more urgent is the problem of continuous monitoring of their state and functioning process. Quality of implementation of functions by component may be affected by number of third-party factors, both internal and external as to the system. Internal influence may be evaluated on the level of subsystems connecting interacting objects. We call this evaluation method interactive [42]. It allows us to determine separate components in selected subsystem, functioning of which is unsatisfactory, without thorough analysis of state and functioning quality of these components and expenses related to such analysis. The simplest interactive evaluation may be performed for system where the movement of flows is deterministic, at least partially, in accordance with certain schedule, the compliance to which may be periodically summed up [1].

In general, only if combined, proposed methods may provide sufficiently full and adequate understanding of CHNS quality. Indeed, high local evaluations do not ensure effective interaction of elements, failures of separate systems components may result in breakdown in balanced organization, satisfactory state of object for the moment of current inspection does not imply the state will stay satisfactory till the next inspection. Huge amount of information regarding separate CHNS elements without appropriate generalization is ill-suited for rapid analysis and timely reaction for drawbacks discovered. On higher generalization levels, evaluation allows to determine reliable conclusion as to the state and operation quality of system and its main subsystems and to define measures, as well as material and finance expenses required for its modernisation and optimization of functioning [40]. At the local level evaluation allows to identify separate elements and their components subject to improvement. These "narrow" places that are constantly discovered during scheduled inspections or continuous system monitoring may be subject to mathematical modelling. This narrows down the object of modelling and makes the process itself more realistic.

\section{Priority of System Components}

The concept of priority of system components and functions that they implement, is important in the study of CHNS [4]. Priority of the component can be defined as a quantitative measure of its significance in the system (in every system there are more and less important objects). In order to reduce the dimension during simulation into the system content and structure first of all are included its highest priority components. In other cases after decomposition of CHNS primary consideration is also given to the study of high-priority objects. The highest priority usually have components, failure of which leads to a failure of the whole system.

Priority of network node can determine its degree and/or betweenness centrality. Among two nodes with the same degree or betweenness centrality the highest priority has the node through which the more flows have movement. The last statement is true for the edges of the network system. Usually, the analysis of the effectiveness of the large scale network system operation can not be carried out simultaneously for all elements (nodes, edges, flows) and components of the higher hierarchy levels. Sometimes the efficiency of certain subsystems of CDS is more important than the operation quality of separate system elements (in human collectives is expressed by the term "team work": an agreed team of "mediocrities" often wins uncoordinated team of "stars"). Routes of flows may be such subsystems in CHNS. The priority of each route is determined by the number and/or volume of flows that pass this route in a certain time period. For flow which moves 
the route is not important priority of node in which it is delayed. This means that from the point of view of analysis of flows movement on the system all the nodes and edges of the route may be considered equally important. Routes can be grouped according to their priority.

\section{Aggregation Methods: the Main Approaches}

The main objective of aggregated evaluation is to create an adequate generalized puttern of the operation quality of system components of various hierarchy levels. Analysis of this puttern can be done by avoiding processing of huge volumes of information that describes the behavior of these components in detail. Aggregated evaluation should significantly simplify and shorten the process of problems localization and preparing of appropriate decisions. Next we analyze the adequacy of generalized conclusions obtained using three different approaches. We illustrate these approaches on the simplest example of a system $S$ that consists of $N$ elements $s_{n}, n=\overline{1, N}$. Let us assume that $e\left(s_{n}\right)$ is evaluation of operation quality of element $s_{n}, e\left(s_{n}\right) \in\left[e^{\min }, e^{\max }\right]$, where $e^{\min }$ and $e^{\max }$ are the minimum and maximum possible evaluations of the operation quality of element $s_{n}$ correspondingly. Methods of formation of multi-criteria and multi-parameter current and forecasting evaluations of behavior of system elements in precise ball scale were described in detail in [30].

Under the first approach the system quality $e^{(1)}(S)$ is determined by the quality of its "weakest" element, i.e.

$$
e^{(1)}(S)=\min _{n=1, N} e\left(s_{n}\right) .
$$

This approach has to use when we evaluate the systems in which the malfunction of elements can lead to failure of the separate subsystems or the whole CDS. An example of such system is the human body in which the unsatisfactory functioning of certain organs can lead to lethal consequences; railway in which the shifts of subgrade may cause a train crash; the gas pipelines in which the result of a crack in the pipe often were explosions with a large number of human victims, etc. Unsatisfactory state of such elements may cause cascading effects [8] that extend in complex networks (massive power outage in regions of the country, spread of epidemics, occurrence of traffic jams, etc.). WEM is one of the main methods for determining the reliability of the technical and information systems. It is an ideal means for evaluation of conveyor systems work. If elements with the lowest evaluations may pose a threat to the functioning of CDS, the elements with the highest evaluations may be a "sample" and used to improve other elements of real systems [21]. Such elements can be used to determine the criteria of practically achievable optimality [27, 43].

Another and the most common approach for obtaining generalized conclusions is a weighted linear aggregation of elements evaluations. In the case of the system considered above, this evaluation is obtained by the ratio

$$
e^{(2)}(S, \boldsymbol{\rho})=<\boldsymbol{\rho}, \mathbf{e}(\mathbf{s})>_{R^{N}} /<\boldsymbol{\rho}, \mathbf{1}>_{R^{N}},
$$

where $\mathbf{e}(\mathbf{s})=\left\{e\left(s_{n}\right)\right\}_{n=1}^{N}, \boldsymbol{\rho}=\left\{\rho_{n}\right\}_{n=1}^{N}$ is a vector of weighted coefficients, which determines the priority of system elements, $\mathbf{1}=\{1\}_{n=1}^{N}$, and $<., .>_{R^{N}}$ is a scalar product in Euclid space $R^{N}$. The main disadvantage of WLAM is neglecting both positive and negative evaluations. Consider the following example. Assume for the production of some device requires supply of $N$ equally important components. Even in the case of delivery $100 \%$ of $(N-1)$ components and $10 \%$ of $N$-th component can be product only $10 \%$ devices. At the same time, evaluation obtained by WLAM when $\boldsymbol{\rho} \equiv \mathbf{1}$, for the scale $\left[e^{\min }, e^{\max }\right]=[0,100]$ gives the value $e^{(2)}(S, \mathbf{1})=100-90 / N$. For large values of $N$, this evaluation is close to $100 \%$, although it is possible to product only $10 \%$ of devices. It is obvious that such evaluation does not correspond to reality. This method is absolutely not fit for evaluating the operation of conveyor systems. 
A more appropriate for the reality results of generalization are obtained using the method of non-linear aggregation. It is based on the following statement [44].

Theorem 1. For an arbitrary set of real numbers $\left\{a_{n}\right\}_{n=1}^{N}, a_{n}>0$, such that $\sum_{n=1}^{N} a_{n}=A$, the maximum value $\prod_{n=1}^{N} a_{n}$ is achieved in the case of $a_{n}=a^{*}=A / N, n=\overline{1, N}$.

If $\left\{a_{n}\right\}_{n=1}^{N}$ is a set of evaluations, this means that the best result among all sets is achieved, when deviation of $a_{n}, n=\overline{1, N}$, from the mean value $a^{*}$ is a minimum. In the case of system $S$ considered above, evaluation of its quality by NAM is obtained by using the ratio

$$
e^{(3)}(S)=\prod_{n=1}^{N} e\left(s_{n}\right) /\left(e^{*}\right)^{N-1}
$$

where $e^{*}=\sum_{n=1}^{N} e\left(s_{n}\right) / N$. The main drawback, which significantly limits the use of the third approach, is the difficulty of taking into account the priority of system elements.

Generalization by means of NAM can be called the rule of "optimality of mediocrity". This rule works wonderfully on a conveyor belt, where from employees require the same productivity. Otherwise conveyor performance is equal to productivity of the "weakest" employee. For normal production requires timely supply of all components, to make the right decision must be synchronized delivery of information flows, etc. However, use this rule, for example, for evaluation of research team work is inexpedient. In this case usually "one person makes a discovery, the second person this discovery confirms (or refutes) and third person "washes the tubes". "Mediocrity" of all is counterproductive here. These considerations determine the areas of potential use of NAM.

Let us assume that $e\left(s_{n}\right)=e^{(1)}(S), n=\overline{1, N}$. Then

$$
e^{(3)}(S)=\frac{\prod_{n=1}^{N} e^{(1)}(S)}{\left(\sum_{n=1}^{N} e^{(1)}(S) / N\right)^{N-1}}=\frac{\left(e^{(1)}(S)\right)^{N} N^{N-1}}{\left(\sum_{n=1}^{N} e^{(1)}(S)\right)^{N-1}}=\frac{\left(e^{(1)}(S)\right)^{N} N^{N-1}}{\left(e^{(1)}(S)\right)^{N-1} N^{N-1}}=e^{(1)}(S) .
$$

Thus, for an arbitrary set of evaluations $\left\{e\left(s_{n}\right)\right\}_{n=1}^{N}$ such that $e\left(s_{n}\right) \geq e^{(1)}(S), n=\overline{1, N}$, we obtain

$$
e^{(3)}(S) \geq e^{(1)}(S) .
$$

Rewrite the aggregated nonlinear evaluation $e^{(3)}(S)$ in the form

and show that

$$
e^{(3)}(S)=\frac{\prod_{n=1}^{N} e\left(s_{n}\right)}{\left(e^{(2)}(S, \mathbf{1})\right)^{N-1}}
$$

$$
e^{(3)}(S) \leq e^{(2)}(S, \mathbf{1})
$$

or

$$
\frac{\prod_{n=1}^{N} e\left(s_{n}\right)}{\left(e^{(2)}(S, \mathbf{1})\right)^{N-1}} \leq e^{(2)}(S, \mathbf{1}) .
$$

From the last inequality we obtain 


$$
\prod_{n=1}^{N} e\left(s_{n}\right) \leq\left(e^{(2)}(S, \mathbf{1})\right)^{N}=\frac{\left(\sum_{n=1}^{N} e\left(s_{n}\right)\right)^{N}}{N^{N}}
$$

or

$$
N^{N} \prod_{n=1}^{N} e\left(s_{n}\right) \leq\left(\sum_{n=1}^{N} e\left(s_{n}\right)\right)^{N} .
$$

The validity of the last inequality follows from the well-known inequality [45]

$$
\sqrt[N]{\prod_{n=1}^{N} a_{n}} \leq \frac{\sum_{n=1}^{N} a_{n}}{N}, a_{n}>0, n=\overline{1, N} .
$$

Furthermore, in the case $e\left(s_{n}\right)=e^{*}$ we obtain

$$
N^{N} \prod_{n=1}^{N} e\left(s_{n}\right)=N^{N} \prod_{n=1}^{N} e^{*}=N^{N}\left(e^{*}\right)^{N}
$$

and

$$
\left(\sum_{n=1}^{N} e\left(s_{n}\right)\right)^{N}=\left(N e^{*}\right)^{N}=N^{N}\left(e^{*}\right)^{N} .
$$

Thus, the next result is in order.

Theorem 2. For arbitrary set of evaluations $\left\{e\left(s_{n}\right)\right\}_{n=1}^{N}, e\left(s_{n}\right) \geq 0, n=\overline{1, N}$, and equally important elements of the system $S$ are fair inequalities

$$
e^{(1)}(S) \leq e^{(3)}(S) \leq e^{(2)}(S, \mathbf{1}),
$$

and $e^{(1)}(S)=e^{(3)}(S)$ if $e\left(s_{n}\right)=e^{(1)}(S)$, and $e^{(3)}(S)=e^{(2)}(S, \mathbf{1})$ if $e\left(s_{n}\right)=e^{*}, n=\overline{1, N}$.

Consider as an example the system $S=\left\{s_{n}\right\}_{n=1}^{N}, e\left(s_{n}\right) \in[0,100], n=\overline{1, N}, N=3,4,5$. Take a limiting case when the evaluation $e\left(s_{1}\right)$ increases uniformly from 0 to 100 and the values $e\left(s_{n}\right)=100, n=\overline{2(1) N}$. We suppose that all system elements have the same priority, i.e. $\boldsymbol{\rho} \equiv \mathbf{1}$. Graphs of behavior of aggregated evaluations obtained using the three approaches described above are shown in Fig. 3. We can see that NAM gives a more adequate and closer to the reality aggregated evaluations for the system of equally important elements than WLAM.

If operation quality of all system elements increases uniformly and simultaneously, the evaluations $e_{3,4,5}^{(2)}$ and $e_{3,4,5}^{(3)}$ are behaving as the evaluation $e_{3,4,5}^{(1)}$. In other words, the evaluations obtained by means of all three approaches converge to the same result, if all values $e\left(s_{n}\right), n=\overline{1, N}$, are approaching to $e^{*}$.

Shown in the Fig. 3 results confirm that the detection and optimization of the "weakest" elements improve quality evaluation of the whole CHNS. The methods discussed above should not be confused with the well-known weighted sum and weighted product methods [46]. These methods are expedient to use for determination of the best or the worst element (alternative) from a given set. In fact, these methods form a relative quality evaluation of one element in comparison with the other system elements. We aim to be based on absolute quality evaluations [30]. 


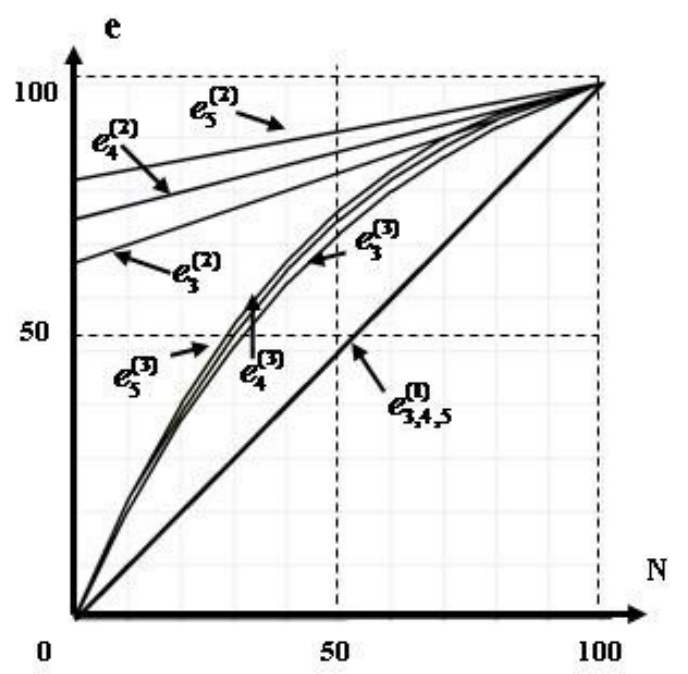

Fig. 3. Generalized conclusions obtained by means of weakest element method $\left(e_{3,4,5}^{(1)}\right)$, weighted linear aggregation method $\left(e_{3,4,5}^{(2)}\right)$ and nonlinear aggregation method $\left(e_{3,4,5}^{(3)}\right)$

\section{Hybrid Aggregation}

It is advisable to combine the approaches described above to get more adequate generalized conclusions. Two ways of such combining can be determined. The first way is to remove the defects detected when using the first approach, and further use the second or third approach. In this case WEM is advisable to apply only to a group of elements with the highest priority.

The relative difference between the evaluations obtained by a combination of first and second or first and third approaches is a quantitative measure of the adequacy of the obtained generalized conclusion. For the above example of the production of some device when $N=3$ and $e\left(s_{1}\right)=10 \%$, $e\left(s_{2}\right)=e\left(s_{3}\right)=100 \%$, the evaluations obtained by WEM, WLAM and NAM in the percentage scale will have the following values: $e^{(1)}(S) \approx 10 \%, e^{(2)}(S, 1) \approx 70 \%, e^{(3)}(S) \approx 20.4 \%$. When first and second approaches are combined the measure of adequacy of aggregated evaluations is determined by the ratio

$$
\sigma_{1,2}(S)=\left(e^{(2)}(S, \mathbf{1})-e^{(1)}(S)\right) / e^{(2)}(S, \mathbf{1}) .
$$

The maximum value of this parameter (equal to 1 ) is achieved with the greatest deviation of aggregated linear evaluation from the evaluation of the "weakest" element and the minimum (equal to 0 ) value is achieved when the evaluations of all system elements are equal. For the last example we have $\sigma_{1,2}(S) \approx 0.857$.

When first and third approaches are combined the measure of adequacy of aggregated evaluations is determined by the ratio

$$
\sigma_{1,3}(S)=\left(e^{(3)}(S)-e^{(1)}(S)\right) / e^{(3)}(S) .
$$

The maximum value of this parameter (equal to 1) is achieved with the greatest deviation of aggregated nonlinear evaluation from the evaluation of the "weakest" element and the minimum (equal to 0 ) value is achieved when the evaluations of all system elements are equal. For the last example we have $\sigma_{1,3}(S) \approx 0.509$. Thus, NAM generates more adequate generalized conclusion than WLAM.

The second way for combination of aggregation methods is as follows. Assume that the elements of system $S$ can be divided into groups $S_{m}=\left\{s_{n}\right\}_{n=1}^{n_{m}}, n_{m} \geq 1, m=\overline{1, M}, \sum_{m=1}^{M} n_{m}=N$. Suppose that all elements of the group $S_{m}$ have the same priority $\widetilde{\rho}_{m}, m=\overline{1, M}$. Then the aggregated evaluation of system $S$ can be done using the ratio 


$$
e^{(4)}(S, \widetilde{\boldsymbol{\rho}})=<\widetilde{\boldsymbol{\rho}}, \mathbf{e}^{(3)}(\mathbf{s})>_{R^{M}} /<\widetilde{\boldsymbol{\rho}}, \mathbf{1}>_{R^{M}},
$$

where $\mathbf{e}^{(3)}(\mathbf{s})=\left\{e^{(3)}\left(S_{m}\right)\right\}_{m=1}^{M}$ and $\tilde{\boldsymbol{\rho}}=\left\{\rho_{m}\right\}_{m=1}^{M}$ is a vector of weighted coefficients, which determines the priority of groups $S_{m}, m=\overline{1, M}$. The last ratio determines a hybrid method for formation of aggregated evaluations that combines WLAM and NAM. It allows to take into account the priority of elements of separate groups (for example, traffic routes of flows) and perform a more adequate evaluation within each group.

From theorem 2 we have that the following statement is in order.

Theorem 3. The next inequalities are fair

where $\boldsymbol{\rho}^{*}=\left\{\left\{\rho_{m}\right\}_{n=1}^{n_{m}}\right\}_{m=1}^{M}$.

$$
e^{(1)}(S) \leq e^{(4)}(S, \widetilde{\boldsymbol{\rho}}) \leq e^{(2)}\left(S, \boldsymbol{\rho}^{*}\right),
$$

If all elements of the system $S$ have a different priority $(M=N)$, the proposed hybrid method is converted into WLAM. If all elements of the system have the same priority $(M=1)$, then it is converted into NAM.

Consider the following example. Assume that the system consists of two groups of elements. Three elements form a first group. Priority of elements of this group is equal to 1.0. The operation quality of the first element increases from 0 to 100, and the operation quality of other two elements of this group is equal to 100. Two elements form a second group. Priority of elements of this group is equal to 0.5 . The operation quality of these elements is equal to 50. Using NAM in this case is not possible, since the elements of the system have different priority. Graphs of behavior of aggregated evaluations, obtained in this case by means of WEM, WLAM and by hybrid method that combines WEM and WLAM are shown in Fig. 4.

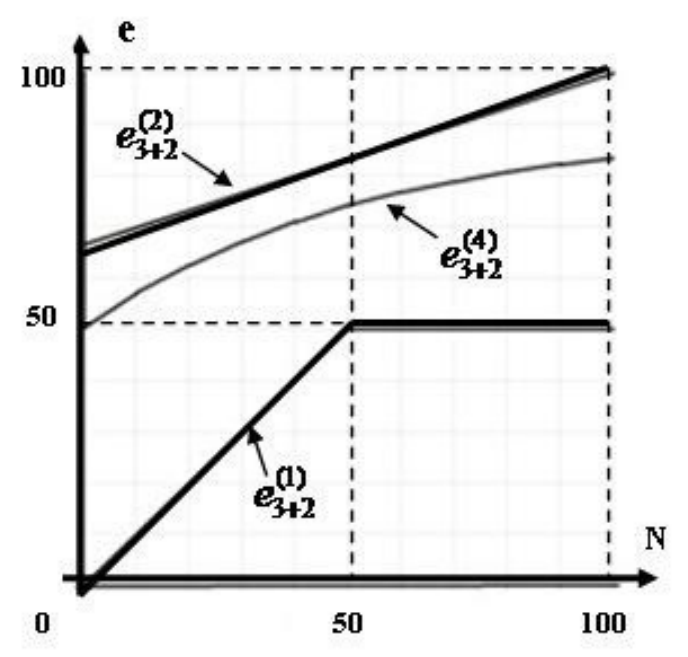

Fig. 4. Generalized conclusions obtained by means of weakest element method $\left(e_{3+2}^{(1)}\right)$, weighted linear aggregation method $\left(e_{3+2}^{(2)}\right)$ and hybrid aggregation method $\left(e_{3+2}^{(4)}\right)$

Note that the operation quality of the elements of the second group is not improved and continues to be equal to 50. Therefore, the aggregated evaluation of the system obtained by WEM is equal to 50 even if the operation quality of elements of the first group is equal to 100 . This is another reason to use WEM only for a set of high-priority elements. 


\section{Conclusions}

We have considered the basic types of network systems and their compound to more global structures: multiplexes, associations and conglomerates. Such systems are actively exchange information flows and generate huge amounts of data which describe their state and operation process. In order to make correct and timely decisions about further actions of the system, these data must be quickly processed and generalized. In general, complex large scale dynamical systems with various structures require different approaches to aggregation. These approaches should ensure maximum adequacy of generalized conclusions [47]. Weighted linear aggregation method does not always guarantee this adequacy, because it can "hide" potentially dangerous components of the system. Weakest element method can be too "radical" and give a negative conclusion about the system quality, based on the quality evaluations of the least important elements. Nonlinear aggregation method is useful if correlation of system component evaluations should be minimal. Thus, all considered above methods have limited areas of application. Using these methods without taking into account the specifics of the system and its separate components can lead to unreliable results of generalization. We propose to use the hybrid approaches for aggregation which should as much as possible to take into account this specificity. The foregoing examples confirm the effectiveness of such approaches.

\section{References}

[1] D. Polishchuk, O. Polishchuk, M. Yadzhak, Complex evaluation of hierarchically-network systems, Automatic Control and Information Sciences. 2 (2014) 32-44.

[2] H. Neng Chiu, The integrated logistics management system: a framework and case study, International Journal of Physical Distribution \& Logistics Management. 25 (1995) 4-22.

[3] T. Berners-Lee et all, World-Wide Web: the information universe, Internet Research. 20:4 (2010) 461-471.

[4] G.A. Marsan, N. Bellomo, M. Egidi, Towards a mathematical theory of complex socioeconomical systems by functional subsystems representation, Kinetic and Related Models. 1:2 (2008) 249-278.

[5] R.G. Rajan, L. Zingales, Financial systems, industrial structure, and growth, Oxford review of economic Policy. 17:4 (2001) 467-482.

[6] A.J. Scott, M. Storper, Regions, globalization, development, Regional studies. 41:S1 (2001) 191-205.

[7] O. Polishchuk, D. Polishchuk, M. Tyutyunnyk, M. Yadzhak, Issues of regional development and evaluation problems, AASCIT Communications. 2:3 (2015) 115-120.

[8] S. Buldyrev et all, Catastrophic cascade of failures in interdependent networks, Nature. 464:15 92010) 1025-1028.

[9] S.R. Weart, Nuclear fear: A history of images, Harvard University Press, 2009.

[10] D.S. Blanchard, W.J. Fabrycky, Systems engineering and analysis (Vol. 4), Englewood Cliffs, New Jersey, Prentice Hall, 1990.

[11] R. Isermann, Fault-diagnosis applications: model-based condition monitoring: actuators, drives, machinery, plants, sensors, and fault-tolerant systems, Springer Science \& Business Media, New York, 2011.

[12] D. Hinrichsen, A.J. Pritchard, Mathematical Systems Theory, Springer, New York, 2005.

[13] S. Boccatti et all, Complex networks: structure and dynamics, Physics Reports. 424 (2006) 175-308.

[14] N.P. Buslenko, Modelling of complex systems, Nauka, Moscow, 1978.

[15] J.T. Allison, Complex system optimization: A review of analytical target cascading, collaborative optimization, and other formulations, Doctoral dissertation, The University of Michigan, 2004.

[16] S. Boccaletti et all, Structure and Dynamics of Multilayer Networks, Physics Reports. 544:1 (2014) 1-122. 
[17] O. Polishchuk, D. Polishchuk, Monitoring of flow in transport networks with partially ordered motion, XXIII Conf. Carpenko physics and mechanics institute, NASU, 2013, 326-329.

[18] C.L. Owen, Evaluation of complex systems, Designe Studies. 28:1 (2007) 73-101.

[19] L. Norros, P. Saviola, Usability evaluation of complex systems, STUK, Helsinki, 2004.

[20] Y. Bar-Yam, About Engineering Complex Systems: Multiscale Analysis and Evolutionary Engineering, Engineering Self-Organising Systems: Methodologies and Applications, Springer, London, 2005.

[21] D. Polishchuk, Polishchuk O., About evaluation of complex dynamical systems, Journal of Complex Systems. 2013, Article ID 204304, 6 p.: Available: http://dx.doi.org/ 10.1155/2013/ 204304.

[22] D. Polishchuk, O. Polishchuk, M. Yadzhak, Comparison of methods of complex system evaluation, Information Extraction and Processing. 32:108 (2010) 110-118.

[23] V.A. Arnold, Catastrophe theory, Springer-Verlag, Berlin, 1992.

[24] J. Dombi, Basic concepts for a theory of evaluation: the aggregative operator, European Journal of Operational Research. 10:3 (1982) 282-293.

[25] A. Wittmuss, Scalarizing multiobjective optimization problems, Mathematical Researches. 27 (1985) 255-258.

[26] O. Polishchuk, Optimization of function quality of complex dynamical systems, Journal of Automation and Information Sciences. 4 (2004) 39-44.

[27] O. Polishchuk, “Optimization of evaluation of man's musculo-sceletal system, Computing Mathematics. 2 (2001) 360-367.

[28] O. Polishchuk, Choice of optimal regimes for functioning of complex dynamical systems, Mathematical Methods and Physicomechanical Fields. 48:3 (2005) 62-67.

[29] O. Polishchuk, About the choice of the optimal dynamical system from the class of equivalent systems, Information Extraction and Processing. 20:96 (2004) 23-28.

[30] D. Polishchuk, O. Polishchuk, M. Yadzhak, Complex deterministic evaluation of hierarchically-network systems: II. Local and forecasting evaluation, System Research and Information Technologies. 2 (2015) 26-38.

[31] A.-L. Barabási, J. Frangos, Linked: the new science of networks science of networks, Basic Books, 2014.

[32] S. Bornholdt, H.G. Schuster, Handbook of Graphs and Networks: From the Genome to the Internet, Jon Wiley \& Sons, 2006.

[33] S.N. Dorogovtsev, J.F.F. Mendes, Evolution of Networks: From Biological Nets to the Internet and WWW, Oxford University Press, 2013.

[34] M. Newman, A.-L. Barabasi, D.J. Watts, The structure and dynamics of networks, Princeton University Press, 2006.

[35] F. Babak, M. Naghmeh, Growing Multiplex Networks with Arbitrary Number of Layers, arXiv:1506.06278v1 [physics.soc-ph], 20 Jun 2015.

[36] D. Polishchuk, O. Polishchuk, M. Yadzhak, Complex deterministic evaluation of hierarchically-network systems: I. Methods description", System Research and Information Technologies. 1 (2015) 21-31.

[37] V. Mayer-Schönberger, C. Kenneth, Big data: A revolution that will transform how we live, work, and think, Houghton Mifflin Harcourt, 2013.

[38] UNESCO Statistical Year-book, 1995.

[39] D. Polishchuk, Evaluation of ukrainian railway equipment condition, Science and Transport Progress. Bulletin of Dnipropetrovsk National University of Railway Transport. 41 (2012) 203-211.

[40] D. Polishchuk, O. Polishchuk, M. Yadzhak, Complex deterministic evaluation of hierarchically-network systems: III. Aggregated evaluation", System Research and Information Technologies. 4 (2015) 20-31.

[41] B.W. Lichtberger, Kostensenkung durch qualitatsvorrat in der fahrweginstandhaltung, Der Eisenbahningenieur. 50:1 (1999) 39-42. 
[42] D. Polishchuk, O. Polishchuk, M. Yadzhak, Complex deterministic evaluation of hierarchically-network systems: IV. Interactive evaluation", System Research and Information Technologies. 1 (2016) 7-16.

[43] O. Polishchuk, Solution of bilateral Dirichlet and Neuman problems for the Laplacian in R3 for tired surface by means of potential theory methods, Applied problems of mechanics and mathematics. 2 (2004) 80-87.

[44] A.D. Polishchuk, About convergence the methods of projections for solution potential theory integral equation, Computer centre of Siberian Division of AS of the USSR, Preprint 776, 1988.

[45] I.N. Bronshtein, K.A. Semendyaev, Handbook of mathematics for engineers and students of technical colleges, Nauka, Moscow, 1986.

[46] D. Strimling, Multi-Criteria Analysis in the Renewable Energy Industry(Green Energy and Technology), Interfaces. 43:2 (2013) 201-202.

[47] D. Polishchuk, O. Polishchuk, M. Yadzhak, Solution of some problems of evaluation of the complex systems, 15th International Conference on Automatic Control, Odesa, Ukraine, 2008, 968-976. 